\title{
AB-INITIO STUDIES OF ALSB (001) ADATOM BEHAVIOR AND RECONSTRUCTION
}

N. A. MODINE *, HANCHUL KIM ${ }^{* *}$, and E. KAXIRAS**

*Sandia National Laboratories, Albuquerque, New Mexico 87185

**Department of Physics, Harvard University, Cambridge, Massachusetts 02138

\section{ABSTRACT}

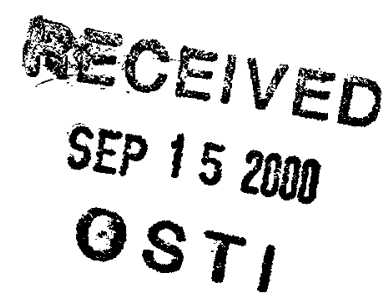

We discuss a recent investigation of adatom behavior on the $\mathrm{AlSb}(001)$ surface using first-principles electronic structure methods based on the density functional theory. For $\mathrm{Al}$ and $\mathrm{Sb}$ adatoms, we find a number of novel adatom structures that differ dramatically from previous results for the superficially similar group-III arsenides. In particular, we conclude that it is energetically favorable for an Al adatom to incorporate substitutionally into the outermost layer of the AlSb surface. This observation helps motivate a proposed new reconstruction for the $\mathrm{AlSb}(001)$ surface. Finally, we argue that the unusual adatom behavior identified for this surface probably results from the presence of a dimer row composed of a double layer of group- $\mathrm{V}$ atoms in the reconstruction, and therefore, it should be generic to all of the antimonides, as well as, the $c(4 \times 4)$ reconstruction of the arsenides and phosphides.

\section{INTRODUCTION}

Epitaxial growth of zincblende III-V semiconductors is the cornerstone of such important technologies as wireless and optical communications and solid state lasers, light emitting diodes, and optical sensors. The nearly lattice matched "6.1 $\AA$ family" consisting of InAs, GaSb, and AlSb shows particular promise for use in high speed electronic and optoelectronic devices. Since such devices typically involve very thin AlSb barrier layers, there is considerable interest in controlling the surface roughness and interface morphology of epitaxially grown AlSb. This requires understanding and controlling the epitaxial growth process. During the epitaxial growth of III-V semiconductors by molecular beam epitaxy (MBE), group-III atoms and group- $\mathrm{V}$ molecules (dimers or tetramers) impinge on the surface. The group- $\mathrm{V}$ species usually stays on the surface for only a short time before returning to the vacuum. In contrast, the group-III species typically sticks and diffuses across the surface. Therefore, it can be argued that the diffusion of group-III adatoms is the principle process controlling MBE growth. Furthermore, understanding the behavior of group-III adatoms is a crucial building block for understanding other growth processes such as island nucleation and step edge attachment. For these reasons, the focus of this paper is an investigation of the behavior of $\mathrm{Al}$ adatoms on the AlSb(001) surface using theoretical techniques based on the Kohn-Sham Density Functional Theory. We will argue that the behavior of $\mathrm{Sb}$ adatoms helps determine the behavior of $\mathrm{Al}$ adatoms, and therefore it will also be discussed. The remainder of the paper is organized as follows: First, we give the background to the present calculations including the experimentally motivated surface reconstruction that we have assumed to be the clean surface. Then, we report details of our method, which are followed by our actual results for the adatom behavior. Finally, we discuss the implications of our method for the stability of our assumed surface reconstruction, and we argue that our results should be generally applicable to a variety of other III-V systems.

\section{BACKGROUND}

Before surface diffusion can be meaningfully studied using first-principles electronic structure techniques, it is necessary to identify the surface reconstruction prevalent under the conditions of interest. Due to the huge configuration space associated with the set of all possible surface reconstructions, it is typically necessary to rely heavily on experimental results (perhaps aided by some calculations) to determine this structure. Under typical conditions used for device growth, the AlSb(001) surface exhibits a $(1 \times 3)$ pattern in diffraction experiments. Based on Scanning Tunneling Microscopy (STM) experiments, Thibado, Bennett, Shanabrook, and Whitman, proposed the structure shown in Fig. 1 as the basic building block of this reconstruction.[1] This structure features raised dimer rows consisting of double layers of $\mathrm{Sb}$ separated by trenches with end-to-end dimers along their centers. There are two different ways in which the second layer $\mathrm{Sb}$ atoms in each trench can be paired to form dimers, and the observed $(1 \times 3)$ diffraction pattern results from incomplete ordering in the way that this pairing occurs in different trenches. Since 


\section{DISCLAIMER}

This report was prepared as an account of work sponsored by an agency of the United States Government. Neither the United States Government nor any agency thereof, nor any of their employees, make any warranty, express or implied, or assumes any legal liability or responsibility for the accuracy, completeness, or usefulness of any information, apparatus, product, or process disclosed, or represents that its use would not infringe privately owned rights. Reference herein to any specific commercial product, process, or service by trade name, trademark, manufacturer, or otherwise does not necessarily constitute or imply its endorsement, recommendation, or favoring by the United States Government or any agency thereof. The views and opinions of authors expressed herein do not necessarily state or reflect those of the United States Government or any agency thereof. 


\section{DISCLAIMER}

Portions of this document may be illegible in electronic image products. Images are produced from the best available original document. 
Figure 1: Top and perspective views of the base AlSb $(2 \times 3)$ structure. The light grey spheres indicate $\mathrm{Al}$ atoms, while the dark grey spheres indicate $\mathrm{Sb}$ atoms. To help show how atoms in one cell of the reconstruction relate to atoms in neighboring cells of the reconstruction, a $(4 \times 6)$ supercell, consisting of four copies of the $(2 \times 3)$ fundamental cell, is shown in each image. The top view shows the outermost three layers of the slab, while six layers are shown in the perspective view. The basic arrangement of atoms was taken from the work of Thibado et al. [1], and the relaxed atomic positions were obtained from a density functional calculation, as described under the METHOD heading.
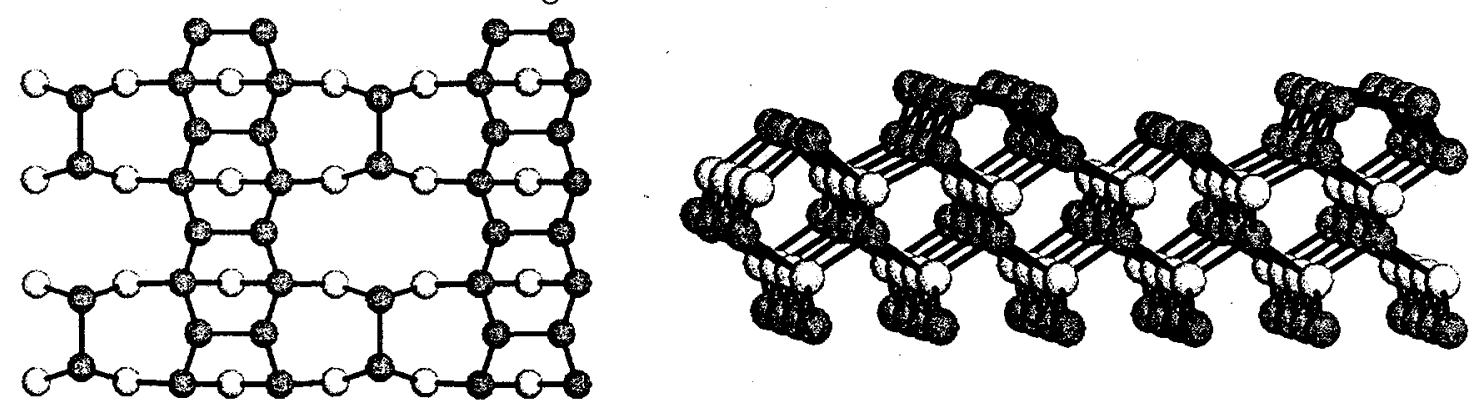

the dimers in different trenches are well separated, we would not expect the intertrench ordering (or lack thereof) to have a significant effect on the adsorption behavior of this surface. Therefore, we choose to study the simplest reconstruction that fully captures the local character of this surface. This is the $(2 \times 3)$ structure, shown in Fig. 1, in which the atoms in every trench are dimerized with the same pattern. A similar structure with the atoms in alternating trenches dimerized in a staggered pattern, has been proposed as the $c(2 \times 6)$ reconstruction observed on $\mathrm{GaSb}(001) \cdot[1,2,3]$

Note that this choice of the $(2 \times 3)$ unit cell divides the dimers in the dimer row into two inequivalent classes: (A) dimers that are centered between dimers in the neighboring trenches, and (B) dimers that are centered between interdimer gaps in the neighboring trenches. For the adatom structures that we discuss below, we find that the average difference in energy between a structure associated with a type-A row dimer and the corresponding structure associated with a type-B row dimer is only $80 \mathrm{meV}$. Since this is considerably smaller than the typical energy difference between structures involving different patterns of local bonding, this confirms our hypothesis that the adsorption behavior of the surface should not depend significantly on the intertrench ordering. Furthermore, since the intention of this paper is to focus on the qualitative behavior of the $\mathrm{AlSb}(001)$ surface, rather than quantitative details, we will treat these two classes of dimers as equivalent throughout the remainder of this paper.

The reconstruction of $\mathrm{AlSb}(001)$ studied in this paper has a couple of unusual features that hint that the results of previous studies[4] of adatom behavior on the $\beta 2(2 \times 4)$ reconstruction of the group-III arsenides may not transfer well to the antimonides. First, $2 / 3$ of the AlSb(001) surface is covered with a double layer of $\mathrm{Sb}$. For the arsenides, such a group-V double layer is only present in the $c(4 \times 4)$ reconstruction observed at atypically high group-V coverages. Secondly, as observed by Thibado et al. [1], this reconstruction does not satisfy the electron counting model (ECM). The ECM is a very simple proposal that has been remarkably successful in predicting the reconstructions of low-index III-V surfaces $[5,6,7,8,9,10,11]$. In essence, the model states that the surface should reconstruct in such a way that the dangling bonds of group-III atoms are unoccupied, and the dangling bonds on group- $V$ atoms are full (i.e. group- $\mathrm{V}$ dangling bonds form lone pairs).

\section{METHOD}

In order to study the AlSb system, we have used the Vienna $A b$ initio Simulation Package (VASP) [12] to perform first-principles electronic structure calculations based on the Kohn-Sham Density Functional Theory (DFT) with plane wave basis sets, ultrasoft pseudopotentials [13], and the local density approximation for the exchange-correlation functional. The DFT is more computationally expensive than empirical 
Figure 2: Top and perspective views of the DFT relaxed AlSb $(2 \times 3)$ structure with an ad-Al in an "Intact Dimer" position on top of the dimer row. The light grey spheres indicate $\mathrm{Al}$ atoms, the dark grey spheres indicate $\mathrm{Sb}$ atoms, and the black spheres indicate ad-Al atoms. Since a $(4 \times 6)$ supercell, consisting of four copies of the $(2 \times 3)$ fundamental cell, is shown, four adatoms are apparent in each view.
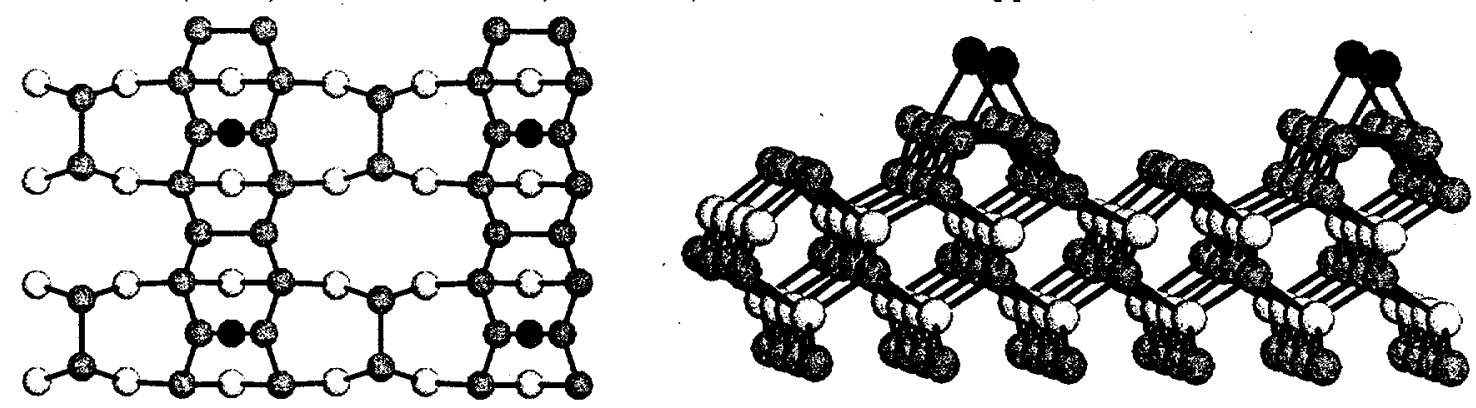

methods, but it gives accurate results for a number of phenomena associated with the AlSb(001) surface that are very difficult to accurately model using empirical techniques. These phenomena include multiple atomic species, interatomic charge transfer, unusual coordinations, and asymmetric bond distributions. Our model of the surface consists of a periodically repeated, asymmetric slab (with six layers of $\mathrm{Al}$ or $\mathrm{Sb}$, and a terminating layer of hydrogen-like atoms) isolated from its images by $19 \AA$ of vacuum. A fictitious $3 / 4$ charged pseudo-hydrogen atom was used to saturate each of the two dangling bonds associated with each back-surface $\mathrm{Sb}$ atom. The two layers next to the hydrogen termination were held at bulk positions, while the rest of the $\mathrm{Al}$ and $\mathrm{Sb}$ atoms were relaxed until each component of the force was less than 0.01 $\mathrm{eV} / \AA$. A plane-wave cutoff of $15 \mathrm{Ry}$ and a k-point sampling equivalent to $6 \mathrm{k}$-points within the $(1 \times 1)$ surface Brillouin zone were used. Adatom behaviors were studied by adding an additional atom at selected locations within the $(2 \times 3)$ unit cell and allowing the system to relax. Since the system (including the extra atom) is periodically repeated in all directions, unphysical interactions between the additional atom and its periodic images are a concern. The slab thickness, $k$-point sampling, and distance between an adatom and its images are only marginally converged in these calculations, but selected tests with up to eight slab layers, $24 \mathrm{k}$-points, and a doubled $(4 \times 3)$ surface supercell have confirmed that qualitative behaviors are accurately determined.

\section{RESULTS}

Figure 2 shows the result of placing an extra $\mathrm{Al}$ atom over the center of a row dimer and relaxing the structure. In this configuration, the dimer bond does not break, and we call this the "Intact Dimer" structure. The bonds between the ad-Al and the dimer atoms are sufficiently short $(2.89 \AA$ vs. $2.66 \AA$ for a bulk AlSb bond) that it is clear that they have a significant covalent character. However, the $\approx 60^{\circ}$ angles of the resulting three membered ring differ dramatically from the $109.5^{\circ}$ angles characteristic of a tetrahedrally bonded semiconductor, indicating a large amount of angular strain. Placing an extra $\mathrm{Al}$ atom over the center of a trench dimer results in a configuration structurally similar to that described above, but $0.5 \mathrm{eV}$ lower in energy. Likewise, two additional structures are obtained by substituting an ad-Sb for the ad- $\mathrm{Al}$ in the above structures and relaxing. Although the structural details change somewhat to reflect the difference in bond lengths, the basic atomic configurations of the ad-Sb structures are the same as the ad-Al structures. In fact, we found this correspondence between ad-Al structures and ad-Sb structures to be a general principle, and throughout the remainder of this paper, we will only mention the exceptions.

Figure 3 shows the result of placing an extra $\mathrm{Al}$ atom into the center of a dimer bond in the dimer row. The atoms that previously formed the dimer are repelled outward until they are too far apart to bond, and we call this the "Broken Dimer" structure. Although there must be a significant energy cost for eliminating the dimer bond, the resulting configuration avoids the highly strained bond angles associated with the Intact Dimer structure. In addition, the ad-Al is pulled down below the level of the row dimers indicating that it is able to further lower its energy by forming partial bonds to $\mathrm{Sb}$ atoms in 
Figure 3: The DFT relaxed AlSb $(2 \times 3)$ structure with an ad-Al in a "Broken Dimer" position on top of the dimer row. Symbols and views are as described in the caption for Fig. 2.
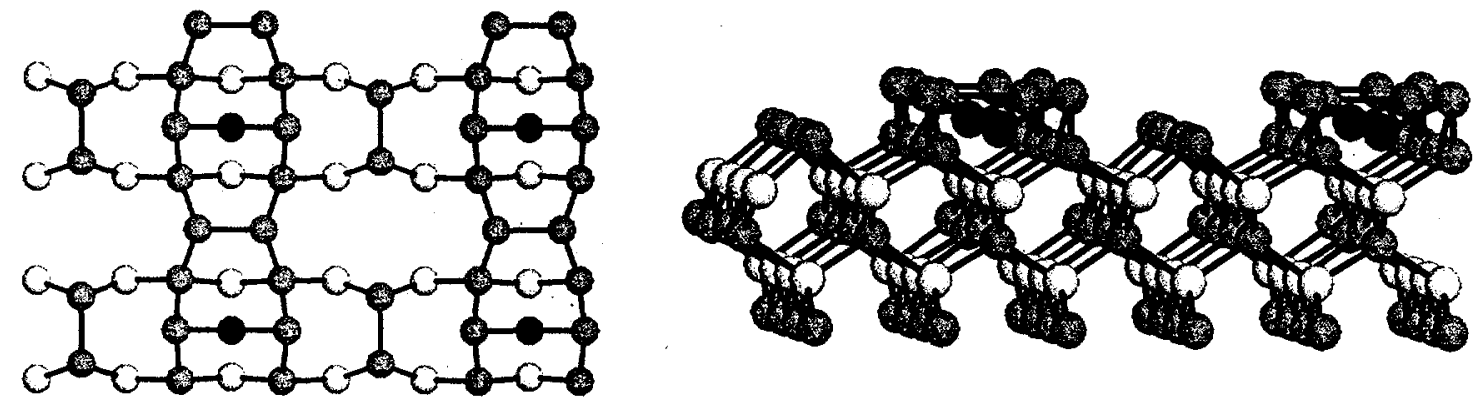

Figure 4: The DFT relaxed AlSb $(2 \times 3)$ structure with an ad-Al in a "Rebonded Broken Dimer" position on top of the dimer row. Symbols and views are as described in the caption for Fig. 2.
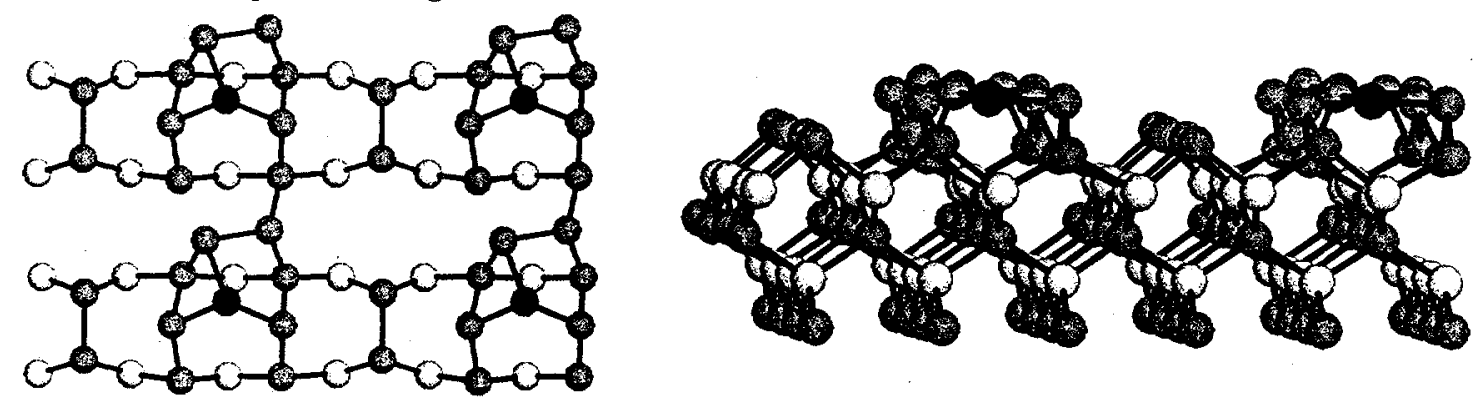

the second layer of the reconstruction. A careful search for a similar structure in which the ad-Al was not pulled into the surface failed to find any such structure. Cumulatively, the energy lowering effects associated with this structure are sufficient to make the Broken Dimer ad-Al structure $1.3 \mathrm{eV}$ lower in energy than the Intact Dimer structure. However, in contrast to the results for the Intact Dimer structure, we were unable to identify a Broken Dimer ad-Al structure associated with the trench dimers. Attempts to create such a structure by pulling the atoms in a trench dimer apart and inserting an extra $\mathrm{Al}$ atom spontaneously relaxed to the Intact Dimer structure. Using an ad-Sb instead of an ad-Al produces two additional structures of the Broken Dimer type. The Broken Dimer ad-Sb structure associated with the dimer row was $0.5 \mathrm{eV}$ lower in energy than the corresponding Intact Dimer structure, but the Broken Dimer ad-Sb structure associated with the trench dimers was slightly (roughly $50 \mathrm{meV}$ ) higher in energy than the corresponding Intact Dimer structure.

These "Intact Dimer" and "Broken Dimer" adsorption sites are similar to those reported by Kley, Ruggerone, and Scheffler for add-Ga [add-Al] atoms on the $\mathrm{GaAs}(001)$ [AlAs(001)] surface. [4] This previous work found that for the $\beta 2(2 \times 4)$ reconstruction of these surfaces, these structures are locally stable structures (i.e. local minima of the total energy). In contrast, for the case of the $(2 \times 3)$ reconstruction of $\mathrm{AlSb}(001)$, we find that these structures are transition states (i.e. saddle points of the total energy).

In particular, breaking the symmetry of the Broken Dimer structure by displacing the adatom by a small amount in an arbitrary direction, and then relaxing the structure, results in the structure shown in Fig. 4. The adatom displaces to the side of the dimer and forms an additional bond to one of the atoms in the neighboring dimer. The neighboring dimer strengthens this bond by twisting so that the atom forming the bond moves closer to the adatom. We refer to this configuration as the "Rebonded Broken Dimer" structure. During the formation of this structure, the twisting of the neighboring dimer weakens one of its back bonds and the bond angles become somewhat more strained. However, a new Al-Sb bond is formed in the process, and the net effect is to lower the energy by $0.7 \mathrm{eV}$ relative to the simple Broken Dimer 
Figure 5: The DFT relaxed AlSb $(2 \times 3)$ structure with an ad-Al in a "Back-Bond" position on top of the dimer row. Symbols and views are as described in the caption for Fig. 2.


structure. A similar rebonding process (without the twisting) allows an adatom bonded to an intact trench dimer to form bonds to the atoms in the neighboring dimer row, lowering its energy by $0.6 \mathrm{eV}$. In the $\mathrm{ad}-\mathrm{Sb}$ case, the corresponding rebonding energies are $0.9 \mathrm{eV}$ and $0.7 \mathrm{eV}$ for the dimer row and the trench, respectively.

Breaking the symmetry of the Intact Dimer structure in a similar fashion produces even more dramatic results. The adatom moves to the side of the dimer and then toward the edge of the dimer row inserting itself into one of the back-bonds of the dimer. The resulting configuration, which we call the "Back-Bond" structure, is shown in Fig. 5. In the process of forming this structure from the Intact Dimer structure, the back-bond is spontaneously broken (No barrier!) as new bonds are formed between the adatom and $\mathrm{Sb}$ atoms in the second layer and in a neighboring dimer. For an ad-Al, the process lowers the energy by $1.9 \mathrm{eV}$, but the final structure is still $0.1 \mathrm{eV}$ higher in energy than the Rebonded Broken Dimer structure discussed above. For an ad-Sb, the energy gain relative to the Intact Dimer structure is $1.4 \mathrm{eV}$, and the final structure is in a virtual tie (a few meV higher in our calculations) with the Rebonded Broken Dimer as the lowest ad-Sb structure that we have found. As might be expected based on the fact that the trench dimer back-bonds are $\mathrm{Al}-\mathrm{Sb}$ bonds rather than $\mathrm{Sb}-\mathrm{Sb}$ bonds, we were unable to find Back-Bond structures associated with the trench dimers.

Despite the fact that the Back-Bond structures come out slightly higher in energy than the Rebonded Broken Dimer structures, the spontaneous breaking of the back-bond suggests that inserting an adatom between the double layers of the reconstruction might be a favorable process. We can continue the bondbreaking process started by the Back-Bond structure by inserting the adatom into two back-bonds of a row dimer atom at the same time. This effectively exchanges the adatom and the $\mathrm{Sb}$ from the row dimer, and we call this type of structure "Exchanged". Since the Sb atom from the dimer row could be ejected into any of the ad-Sb structures identified above (except now one of the dimer row atoms is an Al), there is an entire family of structures of this type. We have thoroughly explored this family with our calculations, and we find that the lowest energy structure has the ejected $\mathrm{Sb}$ in a Rebonded Broken Dimer configuration. The resulting structure is shown in Fig. 6. This Exchanged structure is comparable in energy (about 50 $\mathrm{meV}$ higher in our calculations) with an ad-Al in the Rebonded Broken Dimer configuration.

In our exploration of the possible Exchanged structures, we have assumed that the $\mathrm{Sb}$ atom ejected by the exchange process remains in the same surface unit cell as the $\mathrm{Al}$ atom that replaced it. Since the ejected $\mathrm{Sb}$ atom should be free to diffuse on some time scale, this assumption may not be valid. We can easily explore this possibility by considering the case in which the ejected Sb has diffused sufficiently far away from the $\mathrm{Al}$ atom that the interaction is negligible. In this case, the ejected $\mathrm{Sb}$ atom effectively becomes an ad-Sb atom, while the ad-Al becomes incorporated into the surface by substituting for a $\mathrm{Sb}$ atom in the original $(2 \times 3)$ reconstruction. The latter structure, which we call the "Substituted" structure, is shown in Fig. 7. In order to determine whether it is energetically favorable for a ejected $\mathrm{Sb}$ atom to diffuse away from the exchange site, it is necessary to compare the total energies of two combinations of structures with the same total number of atoms. This comparison is shown in Table I. Conceptually, we can think of diffusing the ejected $\mathrm{Sb}$ from the lowest energy Exchanged structure (that shown in Fig. 6) to a clean $(2 \times 3)$ reconstructed region of the surface. This produces a Substituted region plus a region with an ad-Sb, which we take to be in the lowest energy Rebonded Broken Dimer configuration. The "Atom $\Delta$ " 
Figure 6: The DFT relaxed AlSb $(2 \times 3)$ structure with an ad-Al in an "Exchanged" position in the dimer row. Symbols and views are as described in the caption for Fig. 2.
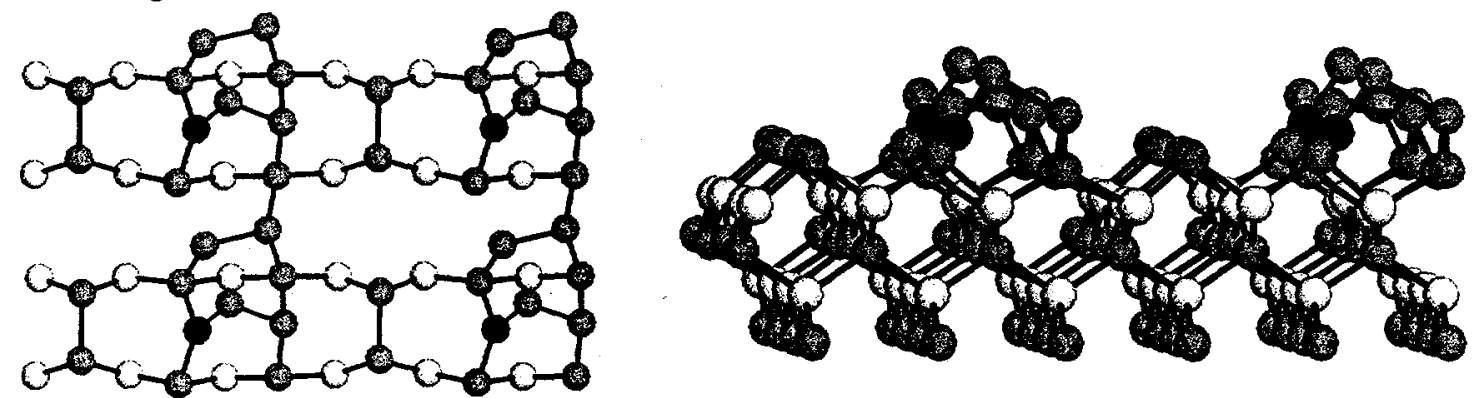

Figure 7: The DFT relaxed AlSb $(2 \times 3)$ structure with an ad-Al in an "Substituted" position in the dimer row. Symbols and views are as described in the caption for Fig. 2.

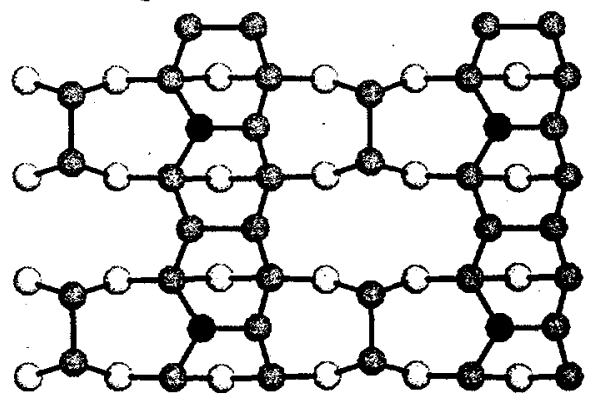

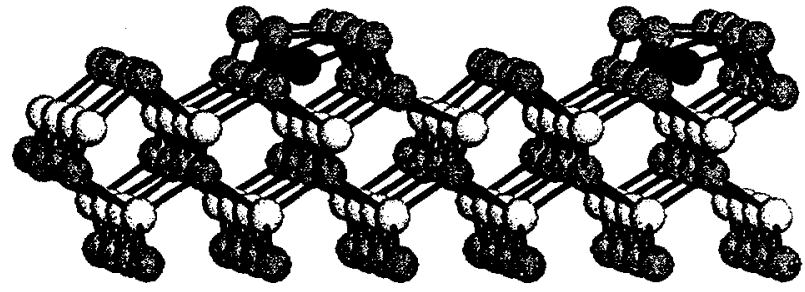

Table I: A comparison of number of atoms relative to the reference $(2 \times 3)$ reconstruction ("Atom $\Delta$ ") and total energies for an ejected $\mathrm{Sb}$ close to and far away from an $\mathrm{Al}$ substituted into the top $\mathrm{Sb}$ layer.

\begin{tabular}{|l|cc|}
\hline \multicolumn{3}{|c|}{ Ejected Sb close to Al Substituted Dimer } \\
\hline Structure & Atom $\Delta$ & Energy (eV) \\
\hline Lowest Exchanged & $+1 \mathrm{Al},+0 \mathrm{Sb}$ & -184.688 \\
Base $(2 \times 3)$ & $+0 \mathrm{Al},+0 \mathrm{Sb}$ & -180.747 \\
\hline Sum & $+1 \mathrm{Al},+0 \mathrm{Sb}$ & -365.435 \\
\hline \hline \multicolumn{3}{|c|}{ Ejected Sb far from Al Substituted Dimer } \\
\hline Structure & Atom $\Delta$ & Energy (eV) \\
\hline Substituted & $+1 \mathrm{Al},-1 \mathrm{Sb}$ & -180.030 \\
Lowest ad-Sb & $+0 \mathrm{Al},+1 \mathrm{Sb}$ & -185.672 \\
\hline Sum & $+1 \mathrm{Al},+0 \mathrm{Sb}$ & -365.702 \\
\hline
\end{tabular}


Figure 8: Top and perspective views of the DFT relaxed AlSb $\beta(4 \times 3)$ structure discussed in Ref. [14]. The light grey spheres indicate Al atoms, while the dark grey spheres indicate $\mathrm{Sb}$ atoms. This structure is obtained from the $(2 \times 3)$ structure by substituting an $\mathrm{Al}$ atom for an $\mathrm{Sb}$ atom in every fourth dimer along the row and shifting the resulting hetero-dimer halfway into the trench. A $(4 \times 6)$ supercell consisting of two copies of the $(4 \times 3)$ fundamental cell is shown in each image.
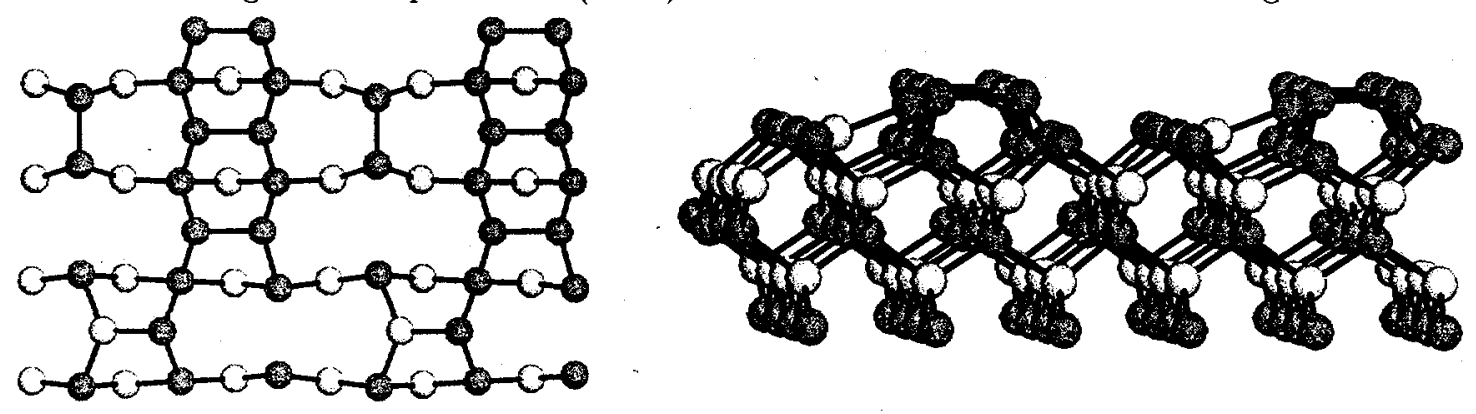

column of Table I gives the change in the number of atoms (relative to the base $(2 \times 3)$ reconstruction) and shows that these two combinations of structures have the same number of atoms. This allows us to directly compare the total energies of the combinations without considering chemical potentials. We find that it is energetically favorable by almost $0.3 \mathrm{eV}$ for the ejected $\mathrm{Sb}$ to diffuse away from the substituted Al. Since entropy also favors separate positions for the ejected $\mathrm{Sb}$ and the substituted $\mathrm{Al}$, we can conclude that, given sufficient equilibration time, an ad- $\mathrm{Al}$ will substitute into the dimer row of the $(2 \times 3)$ reconstruction, creating a new ad-Sb in the process.

\section{DISCUSSION}

The above results indicating that ad-Al atoms substitute spontaneously into the $(2 \times 3)$ reconstruction strongly suggest that the $(2 \times 3)$ reconstruction itself is unstable to some structure involving Al atoms substituted into the dimer rows. A careful investigation of new STM results in concert with theoretical work has confirmed this hypothesis. [14] Figure 8 shows the $\beta(4 \times 3)$ structure identified in Ref. [14] as the stable reconstruction of the $\mathrm{AlSb}(001)$ surface under typical device growth conditions. In addition to an Al substitution into every fourth dimer of the dimer row, this structure involves shifting the Al containing end of these dimers into the trench. This shifting of every fourth dimer allows the structure to satisfy the electron counting model and lowers the energy by almost $0.8 \mathrm{eV}$ relative to a structure with only $\mathrm{Al}$ substitution. As might be expected from our results for ad-Al behavior, Ref. [14] also reports a $\alpha(4 \times 3)$ structure, in which three additional row dimer atoms have been substituted with Al. This structure is shown in Figure 9.

A remaining question is, "Why do we find novel adatom configurations, such as the Rebonded, BackBond, Exchanged, and Substituted structures, for the $(2 \times 3)$ reconstruction of $\mathrm{AlSb}(001)$ when there is no evidence of such structures for the $\beta 2(2 \times 4)$ reconstructions of GaAs(001) and AlAs(001)?" One initially appealing possibility is that these structures result from the failure of the $(2 \times 3)$ reconstruction to satisfy the electron counting model. However; ongoing studies have shown that these unusual adatom structures are equally favored on the $\beta(4 \times 3)$ reconstruction, which does satisfy the $\mathrm{ECM}$, and therefore we can rule out this explanation.

Another possibility is that these structures result from some difference in behavior between arsenic and antimony. In order to investigate this possibility, we have repeated some of our calculations for adatom behavior on the $(2 \times 3)$ reconstruction using an AlAs surface instead of an AlSb surface (although a $(2 \times 3)$ reconstruction of AlAs is not experimentally observed, there is no difficulty in creating such a reconstruction for use in theoretical calculations). The results of this investigation are shown in Table II. Since the energy differences between the ad-Al structures are nearly the same for AISb and AlAs, these results argue that the unusual adatom behavior reported in this paper is primarily a result of the $(2 \times 3)$ reconstruction rather than the AlSb material. The principle feature of the $(2 \times 3)$ (and the $(4 \times 3)$ ) reconstruction that does not occur in the previously studied arsenide reconstructions is the dimer row consisting of a double layer of 
Figure 9: Top and perspective views of the DFT relaxed AlSb $\alpha(4 \times 3)$ structure discussed in Ref. [14]. Symbols and views are as described in the caption for Fig. 8. This structure is obtained from the $\beta(4 \times 3)$ structure by substituting three additional row dimer atoms with $\mathrm{Al}$.
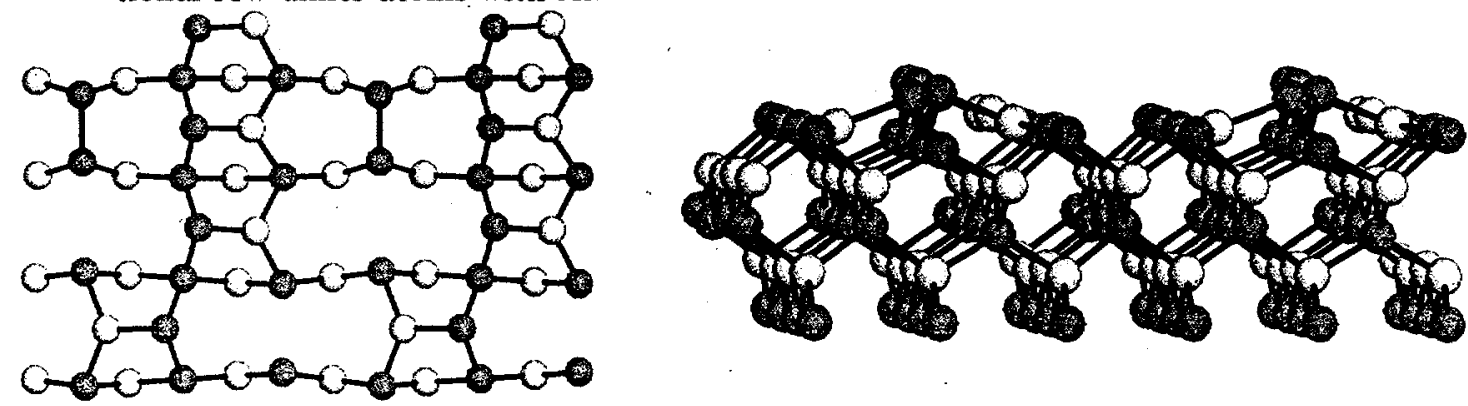

Table II: A comparison of the energies (relative to an unrebonded Broken Dimer configuration) of selected ad-Al structures. All configurations are associated with a row dimer.

\begin{tabular}{|l|cc|}
\hline Structure & AlSb $(2 \times 3)$ & AlAs $(2 \times 3)$ \\
\hline Rebonded Broken Dimer & -0.70 & -0.67 \\
Back-Bond & -0.60 & -0.66 \\
\hline
\end{tabular}


s group-V atoms. Therefore, it is reasonable to speculate that similar novel adatom behaviors (rebonding, back-bond breaking, exchange, and substitution) will occur for all reconstructions that involve a double layer dimer row. This includes not only the (001) surface of AlSb, GaSb, and InSb [14], but also the arsenides and phosphides at high group- $V$ coverages where the $c(4 \times 4)$ reconstruction occurs.

\section{CONCLUSIONS}

We have studies adatom behavior on the $(2 \times 3)$ reconstruction of $\mathrm{AlSb}(001)$ and identified a number of unusual adatom structures. Based on our results, we believe that it is likely that reconstructions featuring a double layer of group- $V$ atoms arranged in a dimer row will generally exhibit similar behavior, including substitutional incorporation of group-III atoms. This conclusion helps support and explain a set of $(4 \times 3)$ reconstructions of III-Sb(001) surfaces that have recently been proposed based on other considerations.

\section{ACKNOWLEDGEMENTS}

This work was supported by NSF and DARPA under the Virtual Integrated Prototyping Initiative, and by a grant of HPC time from the DoD HPC ARL and ASC Major Shared Resource Centers. The $a b$ initio total-energy and molecular-dynamics package VASP (Vienna $A b$ initio Simulation Package) and the corresponding ultrasoft pseudopotential database were developed at the Institute für Theoretische Physik of the Technische Universät Wien. Sandia is a multiprogram laboratory operated by Sandia Corporation, a Lockheed Martin Company, for the United States Department of Energy under Contract DE-AC0494AL85000.

\section{REFERENCES}

1. P. M. Thibado, B. R. Bennett, B. V. Shanabrook, and L. J. Whitman, J. Cryst. Growth 175-176, 317 (1997).

2. M. T. Sieger, T. Miller, and T.-C. Chiang, Phys. Rev. B, 52, 8256 (1995).

3. U. Resch-Esser, N. Esser, B. Brar, and H. Kroemer, Phys. Rev. B, 55, 15401 (1997).

4. A. Kley, P. Ruggerone, and M. Scheffler, Phys. Rev. Lett. 79, 5278 (1997).

5. W. A. Harrison, J. Vac. Sci. Technol., 16, 1492 (1979).

6. D. J. Chadi, J. Vac. Sci. Technol., A5, 834 (1987).

7. M. D. Pashley, Phys. Rev. B, 40, 10481 (1989).

8. C. B. Duke, Surf. Sci., 65, 543 (1993).

9. J. E. Northrup, Phys. Rev. B, 50, 2015 (1994).

10. S. B. Zhang and A. Zunger, Phys. Rev. B, 53, 1343 (1996).

11. C. D. MacPherson et. al., Phys. Rev. Lett., 77, 691 (1996).

12. G. Kresse and J. Hafner, Phys. Rev. B 47, 558 (1993); G. Kresse and J. Hafner, Phys. Rev. B 49, 14251 (1994); G. Kresse and J. Furthmüller, Comput. Mat. Sci. 6, 15 (1996); G. Kresse and J. Furthmüller, Phys. Rev. B 54, 11169 (1996).

13. D. Vanderbilt, Phys. Rev. B 41, 7892 (1990).

14. W. Barvosa-Carter, A. S. Bracker, J. C. Culbertson, B. Z. Nosho, B. V. Shanabrook, L. J. Whitman, Hanchul Kim, N. A. Modine, and E. Kaxiras, Accepted for publication in Phys. Rev. Lett. 